\title{
Efeito do número de aplicações da hCG sobre a resposta ovulatória durante a vida reprodutiva de éguas
}

Gabriela Bertaiolli Zoca, Gabriel de Carli dos Santos, Thiago Martins, Eneiva Carla Carvalho Celeghini, Carla Patricia Teodoro de Carvalho, Renata Lançoni, Thyago Escodro Dercoli, Rubens Paes de Arruda*

Faculdade de Medicina Veterinária e Zootecnia, Universidade de São Paulo (USP), Pirassununga, SP, Brasil

*Autor correspondente

e-mail: arrudarp@usp.br

\section{Resumo}

A inseminação artificial em éguas é otimizada quando fármacos indutores da ovulação são utilizados. A Gonadotrofina Coriônica Humana (hCG), fármaco mais utilizado para tal fim, contribui para a redução do número de serviços durante um ciclo estral, principalmente quando existe uma alta demanda de sêmen do garanhão ou quando o sêmen é criopreservado e limitado. Além disto, diminui custos com transporte, auxilia a sincronização de doadoras e receptoras de embriões, estimula a maturação e ovulação do folículo dominante reduzindo o intervalo interovulatório e estimula a formação do corpo lúteo. Ao ser administrada na presença de um folículo pré-ovulatório com diâmetro $\geq 35 \mathrm{~mm}$ e edema endometrial, a ovulação normalmente ocorre entre 24 e 48 horas após o tratamento com hCG (78 - 91\%). Ainda que confiável, falhas podem ocorrer com maior incidência em éguas idosas, não sabendo ao certo se a falha na indução da ovulação é devido à formação de anticorpos anti-hCG, formados a partir de aplicações prévias do fármaco em uma mesma estação reprodutiva. 0 objetivo deste trabalho foi sustentar a hipótese de que a perda na eficiência da hCG em promover a ovulação não está relacionada às aplicações desta em ciclos consecutivos, mas de que a quantidade de aplicações da hCG ao longo da vida reprodutiva de éguas pode interferir na resposta esperada após sua aplicação. Foram colhidos dados retrospectivos de 122 ciclos estrais de 60 éguas mestiças, Puro Sangue Inglês e Brasileiro de Hipismo, com faixa etária de 2 a 23 anos, ao longo de 10 estações reprodutivas (outubro a março), pertencentes ao Setor de Equideocultura da Prefeitura do Campus Fernando Costa da Universidade de São Paulo, Pirassununga/SP. As éguas foram divididas de acordo com o número de aplicações de hCG realizadas ao longo de suas vidas reprodutivas, sendo que: grupo A, 1 aplicação $(\mathrm{n}=48)$; grupo $\mathrm{B}, 2$ a 3 aplicações $(\mathrm{n}=50)$ e grupo $\mathrm{C}, 4$ a 7 aplicações $(\mathrm{n}=24)$. Foram utilizados 2.500 UI da hCG, por via endovenosa, quando detectado por meio de ultrassonografia modo-B um folículo $\geq 35 \mathrm{~mm}$ de diâmetro e edema endometrial, independentemente de ter sido na mesma estação reprodutiva ou não. A eficiência da hCG na indução das ovulações foi avaliada 48h após sua aplicação, por meio da presença do corpo 
lúteo onde existia um folículo dominante (exame ultrassonográfico modo-B). Para as análises estatísticas utilizou-se o procedimento Glimmix do SAS, versão 9.3. Para as comparações, utilizou-se o teste de médias LSD (opção DIFF do comando LSMEANS). 0 grupo B $(86,0 \% \pm 0,05$ de ovulações) não apresentou diferença significativa $(P>0,05)$ na taxa de ovulação 48 h comparado ao grupo $A(97,9 \% \pm 0,02)$, porém, tendeu a ser menos eficiente. $\mathrm{O}$ grupo $\mathrm{C}$ apresentou eficiência significativamente $(\mathrm{P}<0,05)$ menor que os grupos $\mathrm{A}$ e $\mathrm{B}$ sob a taxa de ovulação em $48 \mathrm{~h}(58,3 \% \pm 0,10)$. Estudos foram realizados ao longo dos anos para determinar a eficiência da hCG após aplicações subsequentes e os resultados são controversos principalmente em torno da formação de anticorpos anti-hCG. Baseando-se em nossos resultados, concluímos que a eficiência da hCG em induzir a ovulação após sua aplicação é comprometida não somente pelo seu uso em ciclos consecutivos, mas também pelo número de vezes em que foi utilizada durante a vida reprodutiva da égua, sendo que o tempo de resposta à ovulação foi significantemente maior em éguas tratadas mais do que quatro vezes.

Palavras-chave: Indução da ovulação. hCG. Anticorpo. 